\title{
MINIATURIZED FERROFLUID ACTUATED SINGLE MODE FIBER VARIABLE OPTICAL ATTENUATOR (VOA)
}

\author{
Anna Duduś, Robert Blue and Deepak Uttamchandani \\ Centre for Microsystems and Photonics, Department of Electronic and Electrical \\ Engineering, University of Strathclyde, Glasgow, UK \\ E-mail: anna.dudus@strath.ac.uk
}

\begin{abstract}
We report a miniature variable optical attenuator employing a ferrofluid actuator/shutter combination located between lensed optical fibers. The VOA consists of an opaque, undiluted ferrofluid plug actuator inside a microfluidic channel. The ferrofluid plug position is translated by a variable magnetic field generated from a miniature electromagnet. The ferrofluid plug actuator in turn displaces a second oil-diluted ferrofluid plug acting as an optical shutter. The position of the shutter controls the optical power coupled between the input and output fibers.
\end{abstract}

\section{INTRODUCTION}

Single mode fiber variable optical attenuators (VOAs) are widely used in fiber optical communications, sensing and signal processing. MEMS based micromechanical VOAs have been widely reported, but these devices have reliability issues associated with movable micromechanical elements [1]. An alternative approach to create a miniature VOA which uses no mechanical moving parts is based on optofluidics technology. Optofluidic based VOAs are exemplified by devices based on liquid crystals [2, 3], variable interfacial reflection [4], electrowetting [5] and the thermo-optic effect [6]. Ferrofluids are a class of fluids that are increasingly being applied in MEMS, mainly in microactuation, since ferrofluid based actuators can operate with low voltage supply, in the 1 to 10 volts range. VOAs incorporating ferrofluids have included translating the ferrofluid along the external surface of an optical fiber long period grating (LPG) to demonstrate a narrow band attenuation up to with up to $6.5 \mathrm{~dB}$ centered at $1580 \mathrm{~nm}$ [7]. More recently, De Pedro et al. [8] reported the use of a ferrofluid doped PDMS cantilever-based VOA, which produced an optical attenuation of up to $3.5 \mathrm{~dB}$ between two multimode fibers as a magnetic field deflected the cantilever. Generally for VOAs used in fiber optic applications, optical attenuation of around $20 \mathrm{~dB}$ over a broadband spectrum is required. In this paper we present a novel ferrofluid based miniature VOA which achieves the above technical specifications.

\section{DEVICE OPERATION}

We report for the first time an "all-fluidic" shutter/actuator, in-line, single-mode fiber VOA with magnetic field based actuation (Figure 1). The shutter is a fluidic shutter and consists of a plug of opaque diluted ferrofluid (comprising a solution of CHE006 ferrofluid in mineral oil in the ratio 1:30) in direct contact with a plug of transparent fluid (water) in a common microfluidic channel. This sliding fluidic shutter is translated by a ferrofluid/magnetic actuator comprising a plug of undiluted oil-based ferrofluid (CHE006) driven by a miniature electromagnet. The ferrofluid plug also sits in the same microfluidic channel, making contact with the sliding fluidic shutter. The shutter is located between a pair of GRIN lensed singlemode fibers positioned on opposite sides of the transparent walled microfluidic channel. By changing the magnetic field produced by the electromagnet, the position of the ferrofluid actuator, and therefore the fluidic shutter, is translated along the channel, thereby varying the optical power transferred between the input and output fibers. In this way, a fiber-optic based VOA is realized.

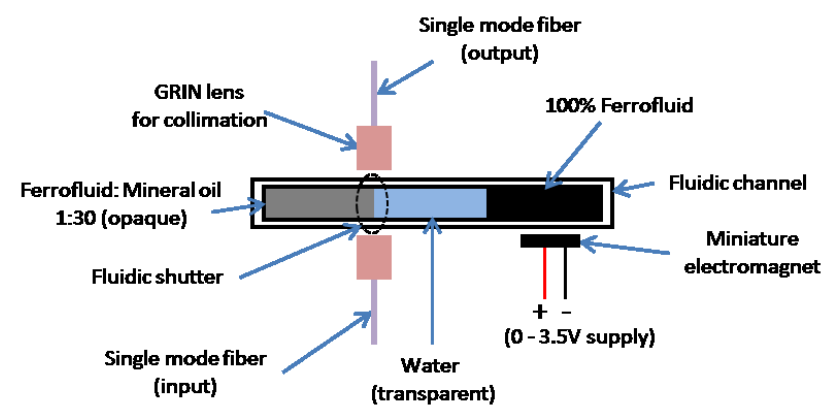

Figure 1. Configuration of our single mode fiber ferrofluid VOA.

\section{THEORETICAL MODEL}

The power distribution of light traveling from the input GRIN lens to the output GRIN lens can be described by a Gaussian function:

$$
A\left(x_{1}\right)=\int_{3 \sigma}^{x_{1}} \frac{1}{\sqrt{2 \pi} \sigma} \exp \left(-\frac{(x-\mu)^{2}}{2 \sigma^{2}}\right)
$$


where $\mathrm{A}\left(\mathrm{x}_{1}\right)$ is the attenuation when an opaque shutter blocks the light from position $3 \sigma$ to position $\mathrm{x}=\mathrm{x}_{1}$. Taking the diameter of the GRIN lens face to be $6 \sigma$ and equal to $1.2 \mathrm{~mm}$ yields a value of $3 \sigma=0.6 \mathrm{~mm}$ while $\mathrm{x}_{1} \epsilon(-3 \sigma, 3 \sigma)$ (Figure 2$)$

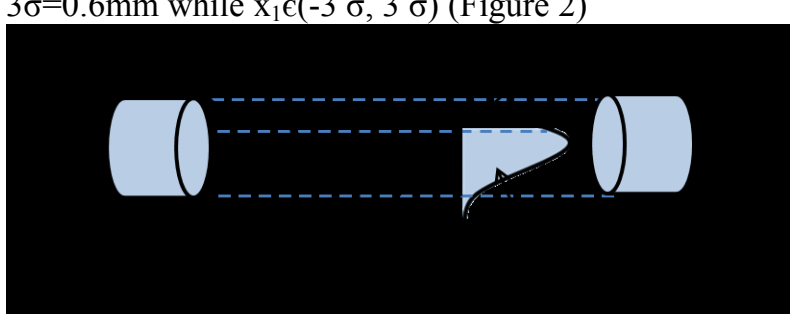

Figure 2. Schematic of Gaussian model system

We have made the assumption that the light power distribution remains constant as light travels between the two GRIN lenses i.e. a parallel beam, and that without the shutter $100 \%$ of the light will be transmitted from one GRIN lens to the other. It is also assumed that the light beam remains circular with diameter of $1.2 \mathrm{~mm}$ (equal to the diameter of the GRIN lens) and that the maximum translation of the shutter $(\mathrm{x}=-3 \sigma)$ will block all the light transmitted between the two GRIN lenses. Table 1 below shows the required shutter displacement across the light beam versus the transmitted optical power.

Table 1. Shutter displacement with corresponding calculated VOA transmission

\begin{tabular}{|c|c|}
\hline Displacement $(\mathrm{mm})$ & Transmission (\%) \\
\hline 1.16 & 0.39 \\
\hline 0.88 & 8.21 \\
\hline 0.72 & 27.56 \\
\hline 0.56 & 58.06 \\
\hline 0.44 & 78.95 \\
\hline 0.36 & 88.63 \\
\hline 0.2 & 97.86 \\
\hline
\end{tabular}

\section{EXPERIMENTAL CHARACTRISATION}

A Santec TSL-210 laser operating at a wavelength of $1550 \mathrm{~nm}$ was used as the optical source. The laser light was coupled into a lensed single mode fiber from where it propagated through the VOA into a second lensed fiber and on to a photodetector/amplifier (LNP-2, Optosci Ltd, UK). The electrical output of the photodetector was recorded by a PicoScope data-logger (Pico Technology Ltd, UK).

Figure 3 shows the change in the optical transmission as the voltage applied to the electromagnet was varied from 0 to 3.5 volts in 0.5 volt steps. The optical transmission changes from $0 \%$ to $100 \%$ transmission (equivalent to approximately a $20 \mathrm{~dB}$ attenuation range). Using this ferrofluid VOA, fine control of the optical transmission between the fibers was achieved. The corresponding position of the ferrofluid shutter within the microfluidic channel can be estimated by comparison with Table 1.

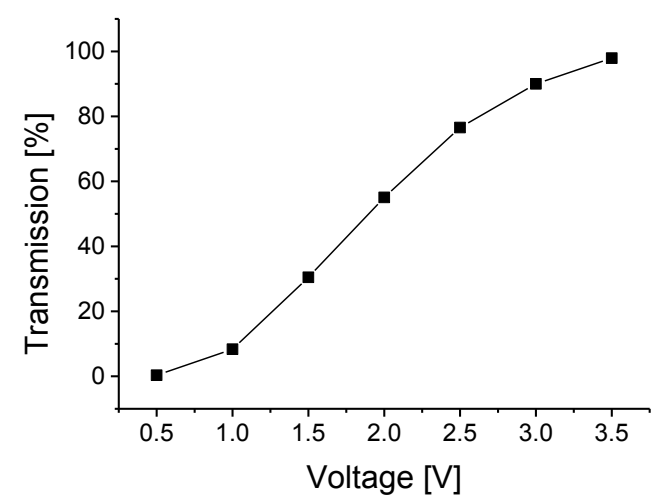

Figure 3: Optical transmission versus actuation voltage to electromagnet.

\section{CONCLUSION}

In conclusion, we have presented a fully fluidic VOA for single-mode fibers using ferrofluid/magnetic actuation. The main advantages of our VOA over previously reported ferrofluidbased VOAs is the simplicity of design, the use of standard single-mode optical fiber, relatively low operating voltage, and the achievement of a higher optical attenuation range which meets typical application requirements.

\section{REFERENCES}

[1] O.Tabata, T. Tsuchiya, O. Brand, G. K. Fedder, C. Hierold J. G. Korvink (eds), Reliability of MEMS: Testing of Materials and Devices (2008), Wiley-VCH.

[2] S. Xu, H. Ren, J. Sun, and S. Wu, Optics Express, 20 (2012), pp. 17059-17064.

[3] J. Liou and C. Yu, OECC 2012, pp. 787-788.

[4] M. I. Lapsley, S. S. Lin, X. Mao, and T. J. Huang, Applied Physics Letters, 95 (2009), p. 083507.

[5] S. A. Reza and N. A. Riza, Optics Communications, 282 (2009), pp. 1298-1303.

[6] I. Martincek and D. Pudis, J. Lightwave Technology, 29 (2011), pp. 2647-2650.

[7] M. Konstantaki, A. Candiani, and S. Pissadakis, ICTON 2009, p. Tu.B4.1.

[8] S. de Pedro, V. J. Cadarso, X. Muñoz-Berbel, J.A. Plaza, J. Sort, J. Brugger, S. Büttgenbach, and A. Llobera, MEMS 2013, pp. 548-51. 